\title{
AVALIAÇÃO DOS INTERPOLADORES KRIGAGEM E TOPO TO RASTER PARA GERAÇÃO DE MODELOS DIGITAIS DE ELEVAÇÃO A PARTIR DE UM "AS BUILT"
}

\section{Evaluation of Kriging and Topo to Raster interpolators for the generation of Digital Elevation Models from a "as built"}

\author{
Edilson José do Carmo ${ }^{1}$ \\ Dalto Domingos Rodrigues ${ }^{1}$ \\ Gerson Rodrigues dos Santos ${ }^{2}$ \\ 1 Universidade Federal de Viçosa - UFV.Programa de Pós-graduação em Engenharia Civil - Informações \\ Espaciais.Viçosa - Minas Gerais - Brasil \\ 2Universidade Federal de Viçosa - UFV.Departamento de Estatística. Viçosa - Minas Gerais - Brasil \\ \{edilson.carmo@ufv.br, dalto@ufv.br, gerson.santos@ufv.br\}
}

\section{Resumo:}

O presente trabalho objetiva realizar o "as built" de uma caixa de decantação de estação de tratamento de água e avaliar o uso dos métodos de interpolação krigagem e Topo to Raster na geração de Modelo Digital de Elevação (MDE) do fundo de uma caixa de decantação de uma Estação de Tratamento de Água levantado com estação total. Para interpolação com o Topo to Raster foram usadas duas metodologias, uma usando como arquivo de entrada linhas de contorno e pontos cotados, conhecido como Topo to Raster padrão, e outra usando somente pontos cotados, conhecido como Topo to Raster condicionado. A interpolação por krigagem foi feita utilizando krigagem ordinária e krigagem simples. Foi utilizado como referência 50 pontos extraídos do MDE gerado pelo método Triangular Irregular Network (TIN). Para cada amostra de discrepância foram calculados indicadores estatísticos. O Modelo gerado por Topo to Raster condicionado apresentou visíveis distorções nas bordas e no centro da área de estudo, devendo ser descartado. Aplicando o teste $\mathrm{z}$, conclui-se que as médias das discrepâncias dos interpoladores krigagem ordinária e krigagem simples não são significativas à um nível de confiança de $95 \%$, ou seja, não diferem do TIN. Já os interpoladores Topo to Raster padrão e Topo to Raster condicionado apresentam diferenças significativas nas médias. Para profundidades médias de 3,21 m, foram encontrados os seguintes valores para os indicadores estatísticos: discrepância média de $1 \mathrm{~cm}$, discrepância máxima de $10 \mathrm{~cm}$, desvio padrão da amostra de $3 \mathrm{~cm}$ e RMS das discrepâncias de $3 \mathrm{~cm}$.

Palavras-chave: Geoestatística, RMS, Caixa de decantação. 


\begin{abstract}
:
This work evaluates the use of Kriging and Topo to Raster interpolation methods in the generation of Digital Elevation Model (DEM) from the bottom of a Water Treatment Station decantation box raised with total station. For the Topo to Raster interpolation two methodologies were used, one using contour lines and quoted points as input file, known as standard Topo to Raster, and another using only quoted points, known as conditioned Topo to Raster. The Kriging interpolation was done using ordinary Kriging and simple Kriging. The DEM generated by the Triangular Irregular Network (TIN) was used as reference. From the isobathymetrics extracted from the DEMs, values were extracted from the interpolated variable and discrepancies were calculated regarding the reference. Statistical indicators were calculated for each discrepancy sample. The model generated by the conditioned Topo to Raster presented visible distortions in the study area edges and center, and must be discarded. Applying the $\mathrm{z}$ test, it is concluded that Kriging and standard Topo to Raster interpolators, applied in small surfaces interpolations, do not differ. For average depths of $3,21 \mathrm{~m}$, the following statistical indicators values were found: average discrepancy of $1 \mathrm{~cm}$, maximum discrepancy of $10 \mathrm{~cm}$, standard sample deviation of $3 \mathrm{~cm}$ and RMS discrepancies of $3 \mathrm{~cm}$.
\end{abstract}

Keywords: Geostatistics, RMS, Decantation box.

\title{
1. Introdução
}

Em uma estação de tratamento de água os decantadores são caixas onde a velocidade da água, após a floculação, sofre uma diminuição para permitir a deposição dos flocos. Geralmente têm formato retangular ou circular. $\mathrm{O}$ fundo tem declividade de acordo com a forma de remoção do lodo (manual ou hidráulica).

O sedimento decantado vai sendo acumulado no fundo da caixa ocorrendo um processo de consolidação. $\mathrm{O}$ acúmulo de grandes quantidades desses sedimentos, afeta de forma substancial o desempenho da caixa de decantação, criando a necessidade de se realizar limpezas periódicas para a retirada dos resíduos. A fim de determinar o volume de resíduos e identificar o momento certo de realizar a limpeza faz-se necessário conhecer, com precisão, o volume da caixa de decantação.

O levantamento dos dados necessários à realização do "as built" pode ser realizado empregando instrumentos como estação total, laser scanner e, em casos de superfícies submersas, ecobatimetros. Entre estes a estação total é, atualmente, o instrumento mais comumente utilizado.

Após a realização de um levantamento topográfico, o produto primário que se tem é uma lista de coordenadas tridimensionais da qual podem-se extrair diversas informações geométricas, entre elas o volume. Uma vez que a lista de coordenadas é uma amostragem discreta da superfície levantada, para uma melhor avaliação do volume, é necessária a geração de uma superfície contínua denominada Modelo Digital de Elevação (MDE), ou seja, é necessária a utilização de métodos de interpolação que utilizam modelos matemáticos para estimar a variável em locais 
não amostrados (Camargo, 1998). O interpolador deve ser o mais acurado possível e gerar uma superfície mais próxima da realidade.

Na base dos métodos de interpolação mais utilizados frequentemente está a primeira lei da Geografia ou Lei de Tobler (1970) que afirma: "Everything is related to everything else, but near things are more related than distant things" que para efeitos de aplicação se pode traduzir como "pontos vizinhos no espaço tem valores mais correlacionados que pontos distantes" (Mesquita, 2009).

Os interpoladores são divididos em duas categorias: os determinísticos e os estocásticos. Os determinísticos geram superfícies considerando apenas as semelhanças espaciais das amostras através de funções matemáticas e não se preocupam com a variabilidade espacial (Burrough e Mcdoneel, 1998). Já os interpoladores estocásticos fazem uso da teoria da probabilidade, e incorporam critérios estatísticos na determinação do peso atribuído aos pontos amostrais para o cálculo das interpolações (Mazzini e Schettini, 2009).

Quando existem dados abundantes, a maioria dos métodos de interpolação produz valores semelhantes e no caso de dados esparsos alguns métodos possuem limitações na representação da variabilidade espacial, porque desconsideram a anisotropia e a continuidade do fenômeno que se quer observar (Burrough e Mcdoneel, 1998).

O presente trabalho tem como objetivo realizar o "as built" de uma caixa de decantação de estação de tratamento de água e avaliar os métodos de interpolação krigagem e Topo to Raster, empregados para gerar o Modelo Digital de Elevação (MDE) do fundo da caixa, comparando-os com o modelo gerado pelo método de interpolação TIN (Triangular Irregular Network). Estes métodos de interpolação vêm se destacando cada vez mais, graças ao avanço computacional.

\section{Geoestatística}

A Geoestatística tem sua base conceitual nos estudos desenvolvidos pelo Matemático e Geólogo Georges Matheron no final da década de 50 e início da década de 60, quando se formalizou a teoria das variáveis regionalizadas a partir dos estudos práticos desenvolvidos por Daniel Krige, no cálculo do volume de reservas de ouro nas minas da África do Sul. Nestes estudos, ele não encontrava sentido nas variâncias estimadas, se não levasse em consideração a distância e a direção da amostragem (Kamimura et al; 2012).

$\mathrm{Na}$ Geoestatística são levadas em consideração características espaciais de autocorrelação de variáveis regionalizadas, onde deve existir uma dependência espacial, fazendo com que os dados amostrados possam ser usados para a estimação de valores nos lugares em que a variável não seja conhecida (Landim, 2000).

A dependência espacial pode ser visualizada a partir de um semivariograma, ou simplesmente variograma. Esta é uma ferramenta fundamental para descrever quantitativamente a variação no espaço de um fenômeno regionalizado (Santos et al; 2011). O estimador do semivariograma é obtido a partir do cálculo das semivariâncias $\hat{\gamma}(\Delta \mathrm{d})$, dadas pela Equação 1: 


$$
\hat{\gamma}(\Delta d)=\frac{1}{2 \cdot N} \cdot \sum_{i=1}^{N}\left[z\left(l_{i}\right)-z\left(l_{i}+\Delta d\right)\right]^{2}
$$

em que $\mathrm{N}$ é o número de pares dos valores $\mathrm{Z}\left(\mathrm{l}_{\mathrm{i}}\right)$ e $\mathrm{Z}\left(\mathrm{l}_{\mathrm{i}}+\Delta \mathrm{d}\right)$, separados por uma distância $\Delta \mathrm{d}, \mathrm{l}_{\mathrm{i}}$ é a posição no sistema de coordenadas e $Z$ é a profundidade observada. Espera-se que as diferenças $\left[\mathrm{Z}\left(\mathrm{l}_{\mathrm{i}}\right)-\mathrm{Z}\left(\mathrm{l}_{\mathrm{i}}+\Delta \mathrm{d}\right)\right]$ decresçam à medida que $\Delta \mathrm{d}$ decresça, ou seja, é esperado que as observações mais próximas espacialmente tenham um comportamento mais semelhante entre si do que aquelas mais distantes. Sendo assim, espera-se que $\hat{\gamma}(\Delta \mathrm{d})$ aumente com a distância (Camargo, 1998; Ferreira, 2012).

Calculando as semivariâncias estimadas para cada $\Delta \mathrm{d}$, é possível verificar o comportamento espacial da variável $\mathrm{Z}$ em estudo, em relação à distância. Este comportamento pode ser visualizado em forma de gráfico ou tabela.

Numa representação gráfica das semivariâncias, como a mostrada na Figura 1, podem ser identificados os seguintes parâmetros (Schaffrath, 2006).

- Alcance: materializa a distância dentro da qual as amostras apresentam-se correlacionadas espacialmente;

- Patamar: é o valor da semivariância correspondente ao seu Alcance (A). A partir deste ponto considera-se que não existe mais dependência espacial entre as amostras, dado que a variação da diferença entre pares de amostras torna-se aproximadamente constante;

- Efeito Pepita: representa a interseção da curva com o eixo $\hat{\gamma}(0)$. Em que o resultado ideal é $\hat{\gamma}(0)=0$. No entanto, para a maioria dos fenômenos estudados existe uma descontinuidade do semivariograma para distâncias menores que a menor distância entre as amostras, então, $\hat{\gamma}(0)$ * O. Parte desta descontinuidade pode ser atribuída à imprecisão da medição, mas é impossível quantificar se a maior contribuição provém desta imprecisão ou da variabilidade de pequena escala não captada pela amostragem (Camargo, 1998).

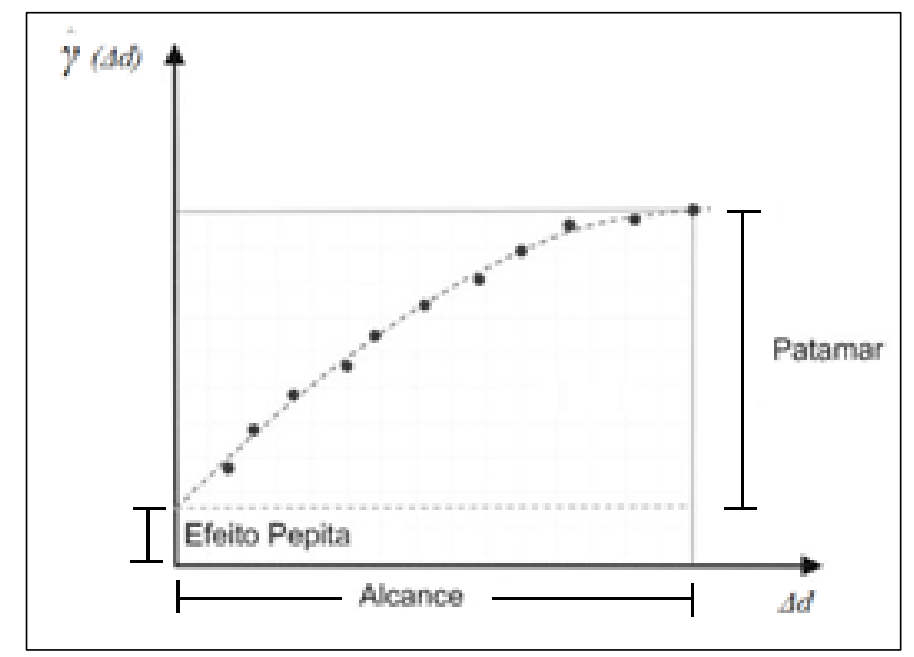

Figura 1: Semivariograma e seus parâmetros. Fonte: (Burrough e Mcdonnel, 1998) adaptado.

Caso exista dependência espacial na variável estudada, pode-se fazer predições em locais não amostrados, por meio de um preditor não tendencioso e de variância mínima, chamado de krigagem (Santos et al; 2011). Segundo os mesmos autores os principais tipos de krigagem linear 
encontrados na literatura são: krigagem simples, krigagem ordinária, krigagem universal e krigagem indicativa.

A principal diferença entre a krigagem e outros métodos de interpolação está na maneira como os pesos são atribuídos a diferentes amostras, sendo determinados principalmente por uma análise espacial baseada no semivariograma experimental. Além disso, uma grande vantagem da krigagem em relação a outros métodos está na possibilidade de obter uma variância de interpolação (Soares, 2006).

A variância de interpolação mede a dispersão local dos pontos amostrados a partir da média ponderada dos quadrados das diferenças entre os valores observados e estimados (Yamamoto, 1999).

\section{Topo to Raster}

O método Topo to Raster foi baseado nos trabalhos desenvolvidos por Hutchinson em seu programa ANUDEM. O êxito desse método se dá por permitir que, além de arquivos de pontos, podem ser usados outros formatos de arquivos como: curvas de nível, delimitações de rios ou lagos, contorno de penhascos e delimitações de bacias, diminuindo, durante o processo de interpolação, os possíveis erros que venham a ser cometidos pelo método (Hutchinson, 1989).

A interpolação dos dados é feita utilizando a soma ponderada dos quadrados dos resíduos a partir dos dados de elevação da superfície representada pela grade amostral (Hutchinson, 2009). Isto é melhor descrito com a definição de um modelo estatístico adequado para os dados, onde o valor de $\mathrm{Z}_{\mathrm{est}}\left(\mathrm{l}_{\mathrm{i}}\right)$ de cada posição $\left(\mathrm{x}_{\mathrm{i}}, \mathrm{y}_{\mathrm{i}}\right)$ é dado pela Equação 2:

$$
\mathrm{Z}_{\text {est }}\left(\mathrm{l}_{\mathrm{i}}\right)=\mathrm{f}\left(\mathrm{x}_{\mathrm{i}}, \mathrm{y}_{\mathrm{i}}\right)+\varepsilon_{\mathrm{i}} \quad \mathrm{i}=1, \ldots, \mathrm{np} \quad
$$

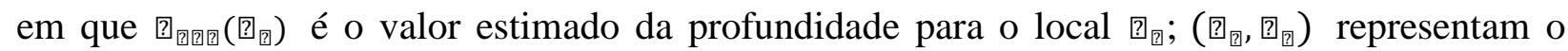
local $l_{i}$ para o qual é estimada a profundidade, $f$ é uma função desconhecida bivariada de amortização para as localizações horizontais em um grid finito, np é o número de pontos amostrados e $\varepsilon_{\mathrm{i}}$ é um resíduo de média zero e desvio padrão $\omega_{\mathrm{i}}$. Assumindo que cada ponto amostral é localizado aleatoriamente entre seu correspondente no grid, o desvio padrão é dado pela Equação 3:

$$
\omega_{\mathrm{i}}=\mathrm{h} \cdot \frac{\mathrm{S}_{\mathrm{i}}}{3,464},
$$

em que h é o espaçamento do grid e $S_{\mathrm{i}}$ é a inclinação de cada célula do grid. A função f é estimada então pela solução que minimiza Equação 4:

$$
\sum_{i=1}^{n p}\left[z_{e s t}\left(l_{i}\right)-f\left(x_{i}, y_{i}\right) / \omega_{i}\right]^{2}+\lambda \cdot J(f),
$$

sendo J(f) uma medida da rugosidade da função $\mathrm{f}$ em termos de primeira e segunda derivadas (Hutchinson, 1989) e $\lambda$ é um número positivo denominado parâmetro de suavização que 
geralmente é escolhido de forma que a soma dos quadrados dos resíduos ponderados na Equação 4 seja igual a np.

Esse método de interpolação foi criado com o objetivo específico de converter dados vetoriais em modelos hidrológicos de elevação de terrenos exatos. O método se utiliza da eficiência computacional da interpolação local, como ponderação do inverso da distância, sem perder a continuidade superficial dos métodos globais de interpolação, como krigagem e spline (Nogueira e Amaral, 2009).

\section{Rede de Triângulos Irregulares (TIN)}

A estrutura TIN utiliza modelo topológico de dados vetoriais, em que as coordenadas planialtimétricas são ligadas por linhas, formando um mosaico de triângulos interconectados de tamanhos variados. A partir das coordenadas planialtimétricas é possível gerar diferentes triangulações. Desta forma, diferentes critérios foram propostos com o objetivo de construir uma triangulação apropriada para interpolações numéricas (Chaves, 2002).

Uma forma de minimizar erros na interpolação é evitar triângulos longos e finos, ou seja, com ângulos agudos. O ideal é que os triângulos sejam o mais equilátero possível. Solução esta encontrada na triangulação de Delaunay que busca maximizar o menor ângulo (Pinto, 1994). Essa triangulação é tão equiângula quanto possível, sendo considerada como padrão para a descrição de superfície (Chaves, 2002).

Em regiões onde há pouca variação de declividade da superfície, os pontos amostrados podem ser mais espaçados, enquanto que em áreas de variação mais intensa na declividade o número de pontos amostrados deve ser maior, de forma que a superfície seja representada com fidelidade. Os pontos devem ser escolhidos de modo a captar as mudanças significativas na forma da superfície, tais como mudanças de declividade.

\section{Materiais e Métodos}

Os dados utilizados neste trabalho foram levantados em uma caixa de decantação da Estação de Tratamento de Água (ETA), da Universidade Federal de Viçosa (UFV). A caixa de decantação possui aproximadamente $20 \mathrm{~m}$ de diâmetro e profundidade média de $3,21 \mathrm{~m}$. O local foi escolhido por apresentar características favoráveis a uma área experimental. Um modelo 3D da caixa é apresentado na Figura 2. 


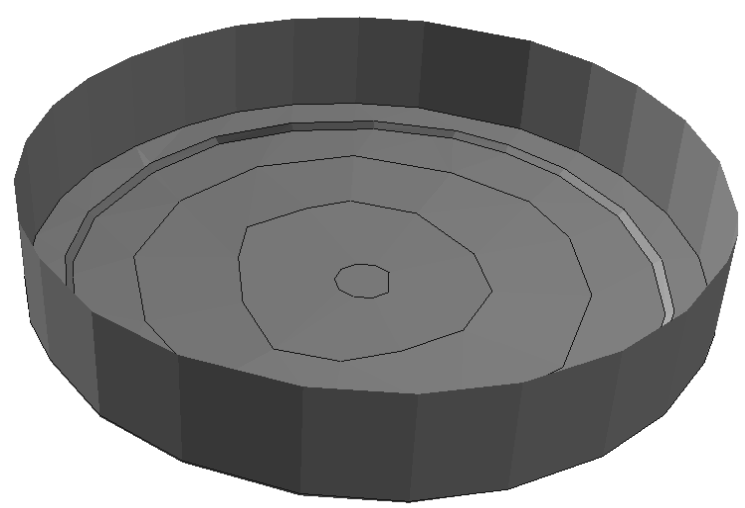

Figura 2: Modelo 3D da caixa de decantação gerado com as observações.

A coleta dos dados foi feita empregando o método irradiação, foram levantados 211 pontos utilizando uma estação total com precisão nominal angular, horizontal e vertical, de 5 segundos e linear de $3 \mathrm{~mm} \pm 3 \mathrm{ppm}$. O levantamento topográfico da borda e do fundo da caixa d'água foi feito no dia da limpeza, com a caixa vazia, levantando todas as feições de interesse para uma melhor representação do fundo. Este levantamento demandou uma avaliação criteriosa da melhor metodologia de levantamento, para que pudesse ser realizado o mais rápido possível após a limpeza, de forma que não se prejudicasse o abastecimento de água. Devido a este fato, não foram realizadas repetições nas observações, o que levaria a uma melhor estimativa dos valores mais prováveis das coordenadas e suas respectivas precisões. Após a coleta, os dados foram processados em um programa desenvolvido no software MATLAB, gerando um arquivo de pontos com coordenadas planialtimétricas $(\mathrm{X}, \mathrm{Y}, \mathrm{Z})$, sendo $\mathrm{Z}$ a profundidade da caixa de decantação contada a partir da borda superior, conforme Figura 3.

As equações empregadas para determinar as coordenadas são:

$$
\begin{aligned}
& \mathrm{X}_{\mathrm{i}}=\mathrm{X}_{0}+\mathrm{D}_{0 \mathrm{i}} \cdot \sin \hat{\mathrm{Z}}_{0 \mathrm{i}} \cdot \sin \mathrm{HZ}_{\mathrm{Oi}}, \\
& \mathrm{Y}_{\mathrm{i}}=\mathrm{Y}_{0}+\mathrm{D}_{0 \mathrm{i}} \cdot \sin \hat{\mathrm{Z}}_{\mathrm{Oi}} \cdot \cos \mathrm{HZ}_{0 \mathrm{i}}, \\
& \mathrm{Z}_{\mathrm{i}}=\mathrm{Z}_{0}+\mathrm{D}_{0 \mathrm{i}} \cdot \cos \hat{\mathrm{Z}}_{\mathrm{Oi}},
\end{aligned}
$$

onde: $\left(\mathrm{X}_{\mathrm{i}}, \mathrm{Y}_{\mathrm{i}}, \mathrm{Z}_{\mathrm{i}}\right)$ são as coordenadas observadas dos pontos levantados; $\left(\mathrm{X}_{0}, \mathrm{Y}_{0}, \mathrm{Z}_{0}\right)$ são as coordenadas arbitradas, e fixas, da estação $0 ; \mathrm{D}_{0 \mathrm{i}}$ é a distância inclinada entre a estação 0 e o ponto i; $\hat{Z}_{0 i}$ é o ângulo zenital de 0 para i e $\mathrm{HZ}_{0 \mathrm{i}}$ é o azimute de 0 para $\mathrm{i}$.

Para as coordenadas $\mathrm{X}_{0}, \mathrm{Y}_{0}$ e $\mathrm{Z}_{0}$ foram atribuídos os valores 1000,1000 e $0,75 \mathrm{~m}$. O valor de $\mathrm{Z}_{0}$ foi atribuído tomando a borda da caixa d'água como referência. 


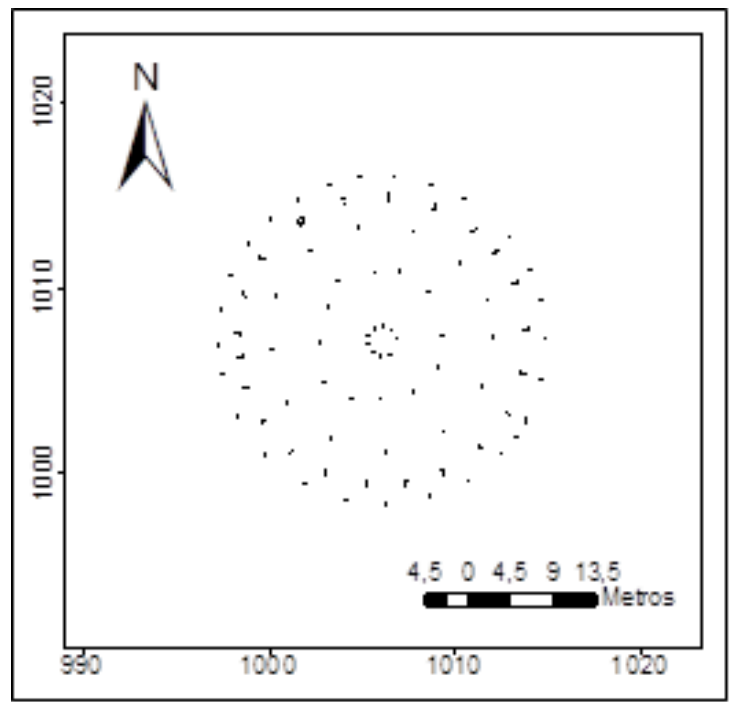

Figura 3: Localização dos Pontos Amostrados.

Como não foi possível a repetitividade das observações, as incertezas nominais do instrumento empregado foram propagadas para as coordenadas determinadas aplicando a lei de propagação das variâncias às Equações 5, 6 e 7.

A coordenada Z, usada como variável a ser interpolada, foi submetida a uma análise exploratória.

A análise exploratória de dados nos fornece um extenso repertório de métodos para um estudo detalhado dos dados, antes de utiliza-los.

Além da construção de tabelas e gráficos, a análise exploratória de dados, consiste também de cálculos de medidas estatísticas que resumem as informações obtidas dando uma visão global dos dados.

Com a análise exploratória pôde-se obter a estimativa da média, variância, desvio-padrão, valor máximo, valor mínimo das profundidades medidas além de permitir detectar possíveis outliers (valores que apresentam grande afastamento em relação aos demais, considerados valores “anormais" ou erros grosseiros) (Guimarães, 2004; Vilela, 2004).

Em seguida, empregando o software ArcGis 10, as profundidades foram interpoladas utilizando os métodos krigagem, Topo to Raster e TIN para a geração dos MDEs.

$\mathrm{Na}$ interpolação utilizando krigagem foram gerados dois MDEs: o primeiro aplicando a krigagem ordinária e o segundo aplicando a krigagem simples. A principal diferença entre elas é que, na krigagem simples assume-se a função média do processo estocástica conhecida, já na krigagem ordinária não precisa conhecer esta média (Andriotti, 1989).

Antes deve ser realizada uma análise geoestatística com o objetivo de modelar a dependência espacial da variável a ser interpolada através do ajuste de modelos teóricos aos semivariogramas experimentais (Santos et al; 2011).

Caso seja constatada a presença de dependência espacial entre os dados, pode-se aplicar o método de krigagem para predizer a variável em estudo nos locais não amostrados a partir dos dados medidos, de acordo com a Equação 8 (Camargo, 1998; Mesquita, 2009). 


$$
\mathrm{z}_{\mathrm{est}}\left(\mathrm{l}_{\mathrm{i}}\right)=\sum_{\mathrm{i}=1}^{\mathrm{np}} \mathrm{p}_{\mathrm{i}} \cdot \mathrm{z}_{\mathrm{i}},
$$

em que $\mathrm{p}_{\mathrm{i}}$ são os pesos associados a cada valor $\mathrm{Z}_{\mathrm{i}}$.

Na interpolação por Topo to Raster foram gerados dois MDEs: o primeiro aplicando a função aqui denominada "Topo to Raster padrão", utilizando como arquivo de entrada pontos e linhas de mudança de declividade, e o segundo, denominado "Topo to Raster condicionado", utilizando como entrada somente arquivos de pontos. Este segundo processo foi realizado pelo fato de a interpolação por krigagem ser feita somente com pontos e desta forma pode-se analisar os interpoladores nas mesmas condições.

$\mathrm{Na}$ interpolação TIN foram utilizados, como dados de entrada, pontos e linhas de mudança de declividade, buscando uma melhor representação da superfície do fundo da caixa d'água. A utilização deste modelo como referência, se deu pelo fato de sua característica construtiva utilizar pontos e isolinhas levantados nas mudanças de declividade (Chaves, 2002).

Após a geração dos MDEs foram extraídas curvas isobatimétricas resultantes da aplicação dos interpoladores. Das isobatimétricas da interpolação TIN foram extraídos 50 pontos para serem usados como referência no cálculo das discrepâncias em relação aos outros métodos conforme Equação 9. Essa quantidade de pontos foi escolhida baseada na norma Americana NSSDA, que diz que cerca de 20 pontos distribuídos por toda área é o suficiente. Por motivo de segurança, devem-se utilizar mais pontos do que é recomendado pela norma (Santos, 2010).

O TIN foi utilizado como referência por ser um interpolador exato. Esses geralmente são utilizados quando se tem certeza dos valores dos pontos no qual a interpolação está baseada. Eles sempre honram os dados, de maneira que após o processo de interpolação não há presença de resíduos, ou seja, a predição sobre os locais amostrados vai ser igual ao próprio valor amostrado (Mazzini e Schettini, 2009).

$$
\mathrm{d}_{\mathrm{i}}=\mathrm{Z}_{\text {est }}\left(\mathrm{l}_{\mathrm{i}}\right)-\mathrm{Z}_{\mathrm{TIN}}\left(\mathrm{l}_{\mathrm{i}}\right) \text {, }
$$

Para análise estatística das discrepâncias foram calculados os seguintes índices: a média, o desvio padrão e a raiz da média dos quadrados das discrepâncias ( $\mathrm{RMS}_{\mathrm{d}}$ ) empregando as Equações $(10,11$ e 12$)$ respectivamente:

$$
\begin{aligned}
& \overline{\mathrm{d}}=\frac{\sum_{i=1}^{n d} \mathrm{~d}_{\mathrm{i}}}{\mathrm{nd}}, \\
& \mathrm{Sd}=\sqrt{\frac{\sum_{\mathrm{i}=1}^{\mathrm{nd}}\left(\mathrm{d}_{\mathrm{i}}-\overline{\mathrm{d}}\right)^{2}}{\mathrm{nd}-1},} \\
& \mathrm{RMS}_{\mathrm{d}}=\sqrt{\frac{\sum_{\mathrm{i}=1}^{\mathrm{nd}}\left(\mathrm{d}_{\mathrm{i}}\right)^{2}}{\mathrm{nd}-1},}
\end{aligned}
$$

onde: nd é o números de discrepâncias na amostra; $\mathrm{Z}_{\mathrm{TIN}}\left(\mathrm{l}_{\mathrm{i}}\right)$ é ovalor da profundidade retirado da isobatimétrica gerada pelo TIN para o local $1_{i}$; $\bar{d}$ é a média das discrepâncias e Sd é o desvio padrão da amostra de discrepâncias. 
Aplicando as Equações (9, 10, 11 e 12), esperam-se amostras, de discrepâncias, coesas e com média nula. Para a krigagem o $\mathrm{RMS}_{\mathrm{d}}$ diminui se o modelo adotado para o semivariograma teórico for bem escolhido (Santos et al; 2011).

\section{Resultados e Discussão}

Os resultados da propagação das incertezas do levantamento topográfico mostraram que as precisões médias para as coordenadas $\mathrm{X}, \mathrm{Y}$ e $\mathrm{Z}$ foram, respectivamente, 0,$003 ; 0,003$ e 0,001 metros.

$\mathrm{Na}$ análise exploratória da variável Z, a ser interpolada, chegou-se aos resultados da Tabela 1.

Tabela 1: Análise exploratória da variável Z (Profundidades).

\begin{tabular}{l|l}
\hline Média $(\mathrm{m})$ & 3,210 \\
\hline Variância $\left(\mathrm{m}^{2}\right)$ & 0,040 \\
\hline Desvio Padrão (m) & 0,206 \\
\hline Profundidade Máxima (m) & 3,553 \\
\hline Profundidade Mínima (m) & 2,980 \\
\hline
\end{tabular}

Empregando o método boxplot, não foram encontrados "outliers" na amostra, o que poderia afetar o resultado da interpolação.

De acordo com os valores da Tabela 1, verifica-se que a profundidade média é de $3,21 \mathrm{~m}$, variância de $0,040 \mathrm{~m}$, desvia padrão de $0,206 \mathrm{~m}$, profundidade máxima de $3,553 \mathrm{~m}$ e profundidade mínima de $2,980 \mathrm{~m}$.

O MDE gerado pelo TIN pode ser visto na Figura 4. 


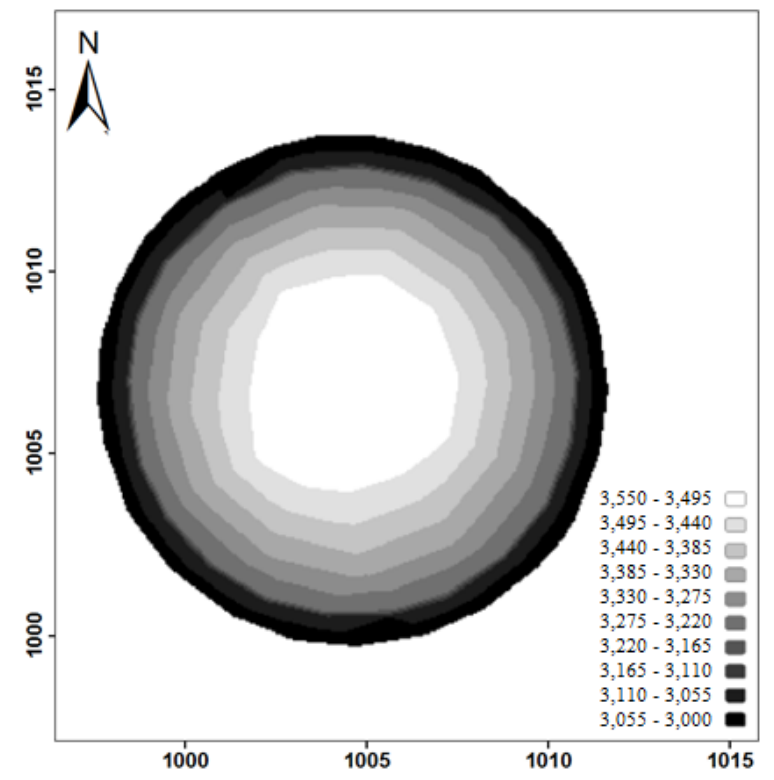

Figura 4: MDE gerado pelo TIN.

$\mathrm{Na}$ análise geoestatística, feita a partir do semivariograma experimental, foi detectada a existência de dependência espacial nos dados conforme Figura 5. Dos principais modelos teóricos existentes, destacam-se o modelo exponencial, o modelo esférico e o modelo gaussiano (Carvalho, 2012). Estes modelos foram submetidos em uma análise de auto validação, onde o melhor que se ajustou ao semivariograma experimental foi o gaussiano.

O modelo gaussiano é um modelo transitivo, em que apresenta um crescimento lento da função do variograma, com o comportamento parabólico na origem. É muito utilizado para modelar fenômenos extremamente contínuos e regulares (Isaaks e Srivastava, 1998).

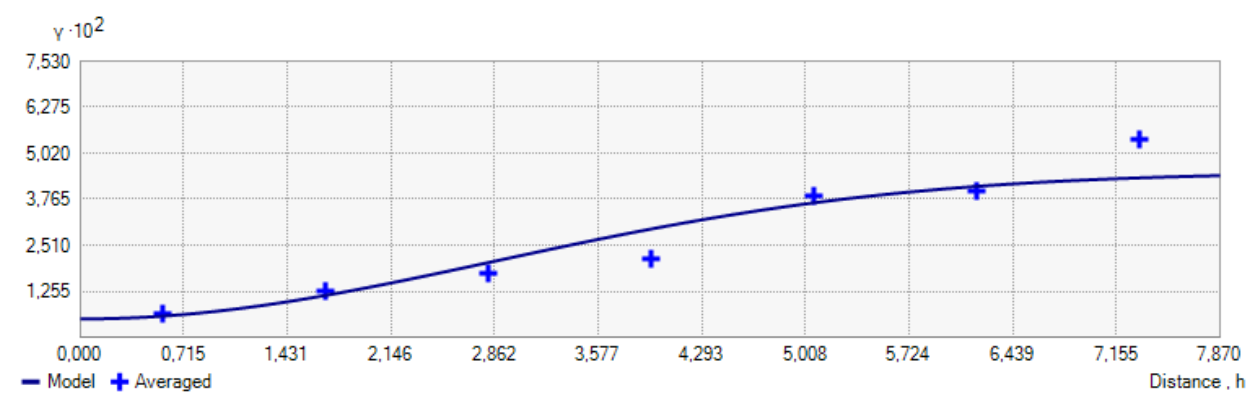

Figura 5: Semivariograma experimental e modelo gaussiano ajustado às semivariâncias estimadas.

Os valores dos parâmetros do semivariograma experimental foram: efeito pepita de $0,005 \mathrm{~m}$; patamar de $0,45 \mathrm{~m}$ e alcance de $7 \mathrm{~m}$.

Após a verificação de dependência espacial nos dados aplicou-se a krigagem ordinária e krigagem simples para predizer a variável em estudo, coordenada Z, para locais não amostrados 
conforme sugere Landim et al (2002). Gerando-se então o MDE da krigagem ordinária e o MDE da krigagem simples (Figura 6) e suas respectivas isobatimétricas (Figuras 7).
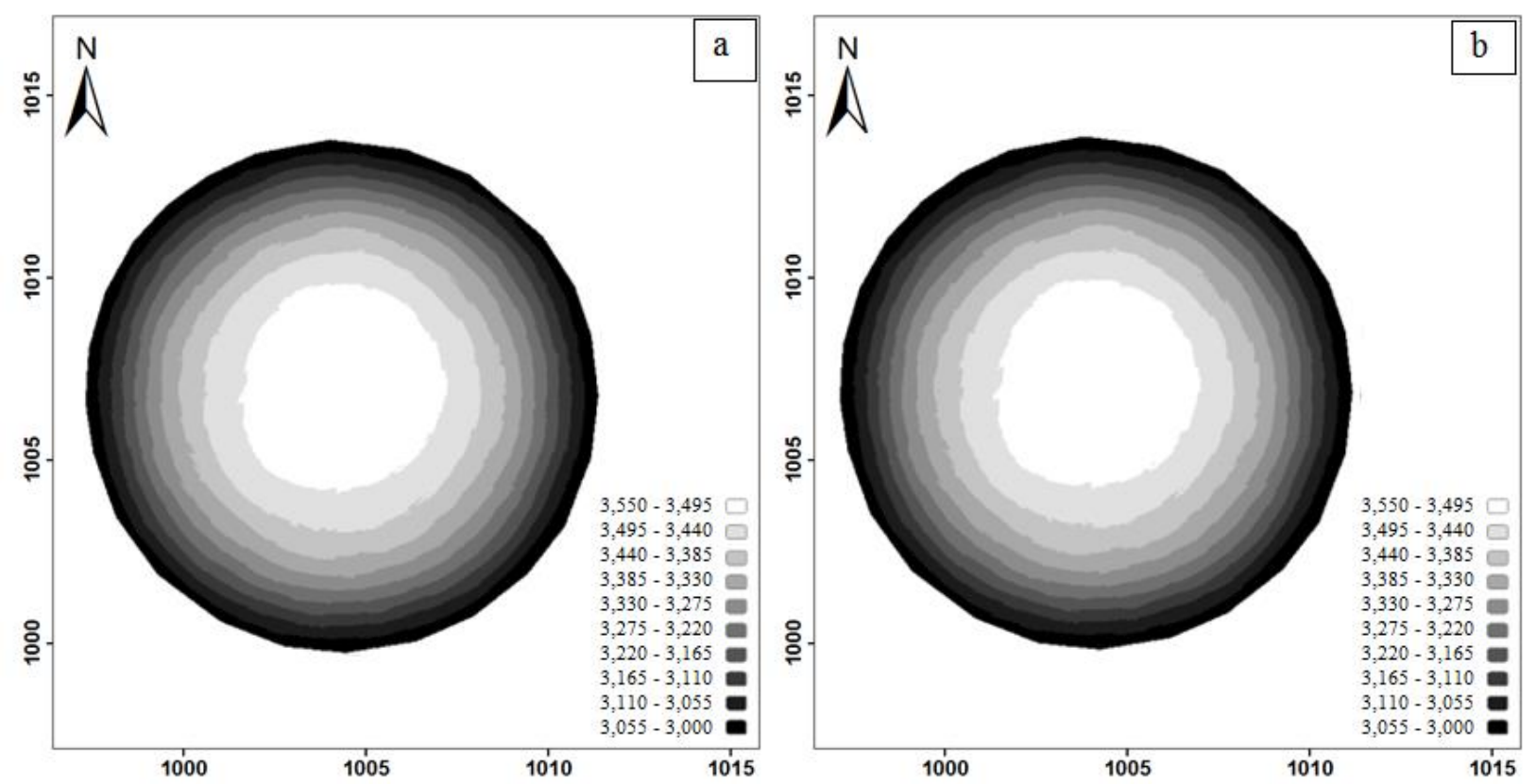

Figura 6: MDEs gerados pela krigagem ordinária (Figura 6-a) e pela krigagem simples (Figura 6-b).
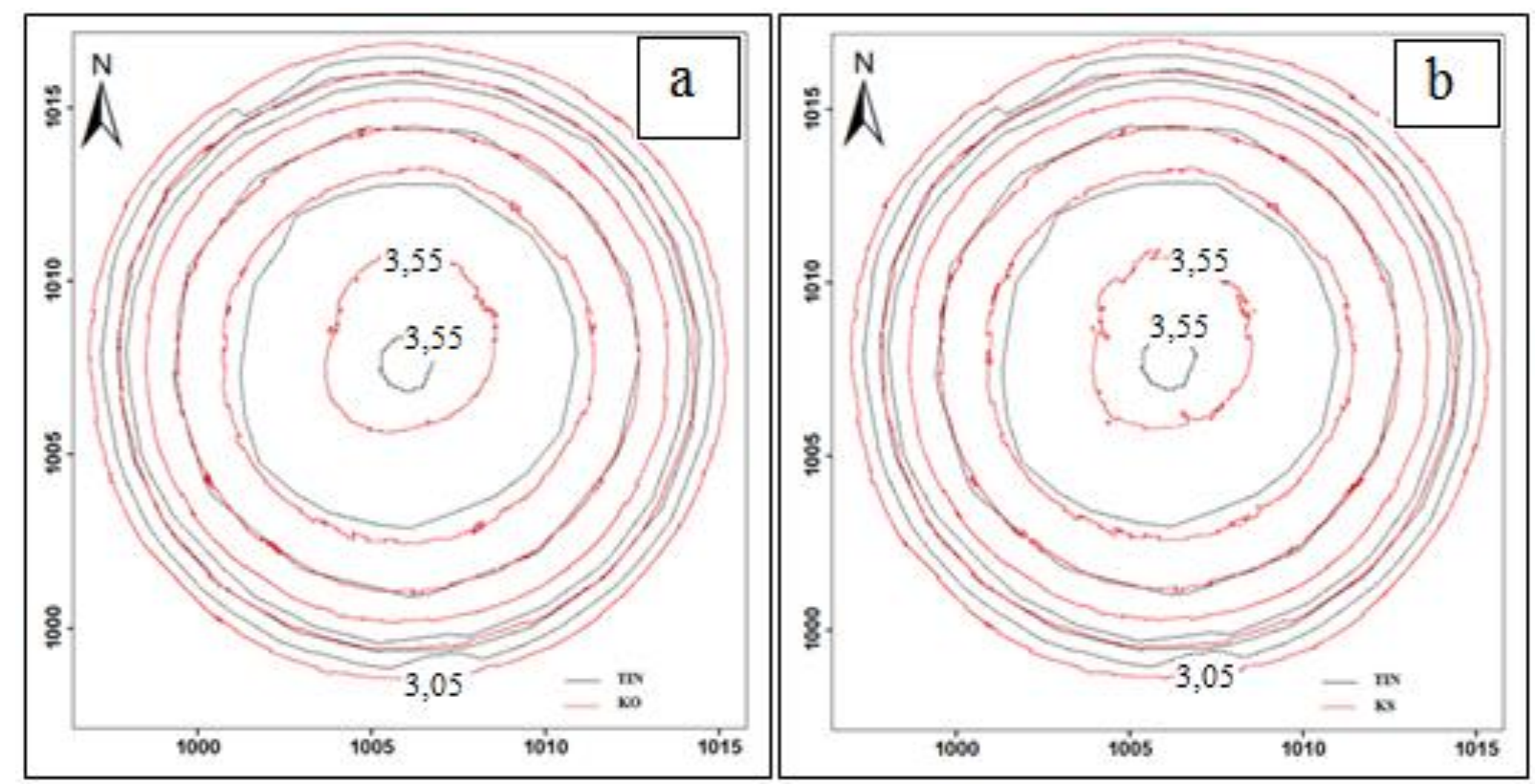

Figura 7: Na cor preta, isobatimétricas geradas pelo método TIN e em vermelho, isobatimétricas geradas pela krigagem ordinária (Figura 7-a) e pela krigagem simples (Figura 7-b). 
Analisando as isobatimétricas na Figura 7, verifica-se uma maior diferença entre as isobatimétricas geradas pelos métodos TIN e krigagem, na região central. Observa-se nas Figuras 4 e 6 que a região central possui uma mesma profundidade.

Os MDEs gerados utilizando Topo to Raster padrão e Topo to Raster condicionado são apresentados na Figura 8 e suas respectivas isobatimétricas na Figura 9.
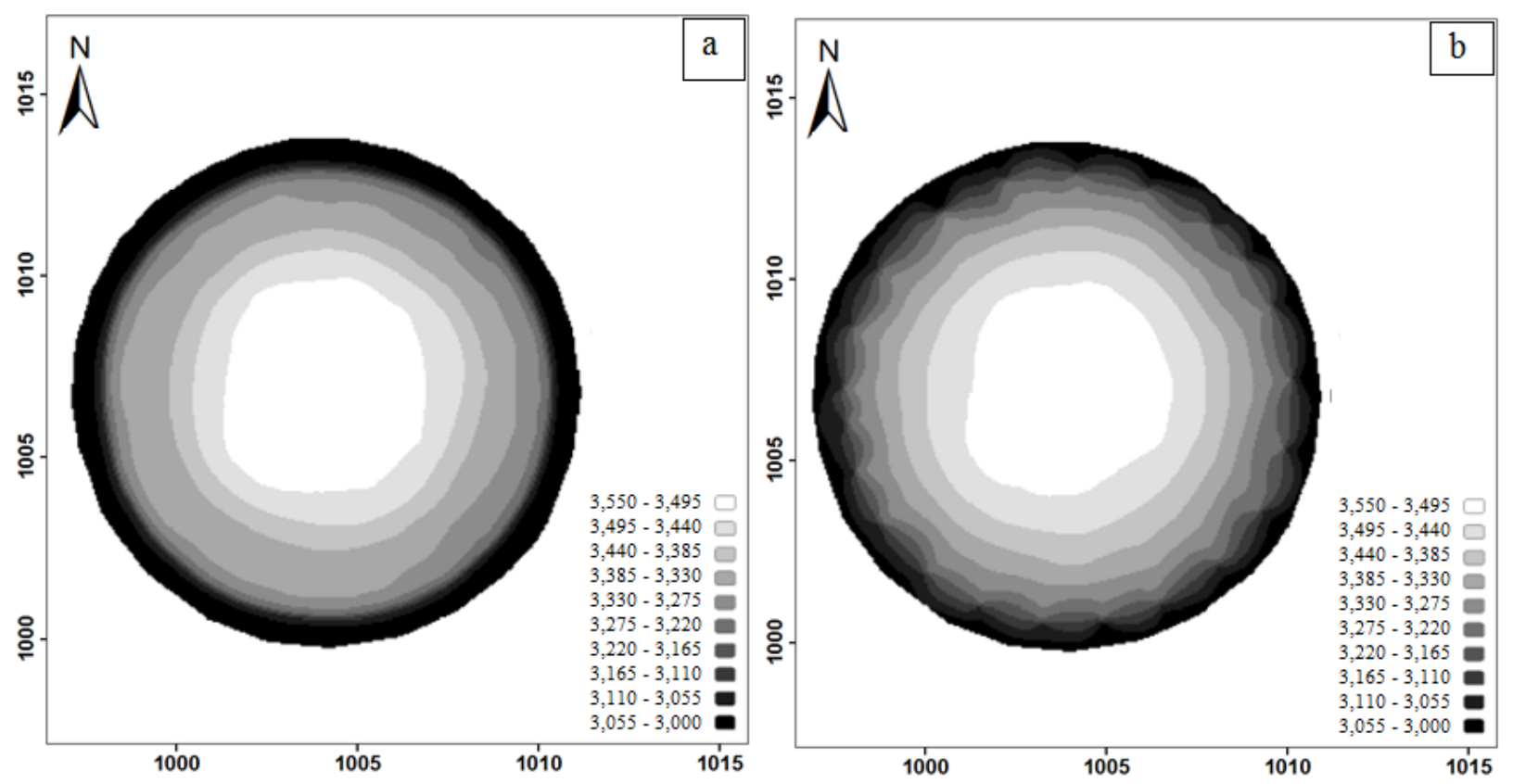

Figura 8: MDEs gerados pelo Topo to Raster padrão (Figura 8-a) e pelo Topo to Raster condicionado (Figura 8-b).
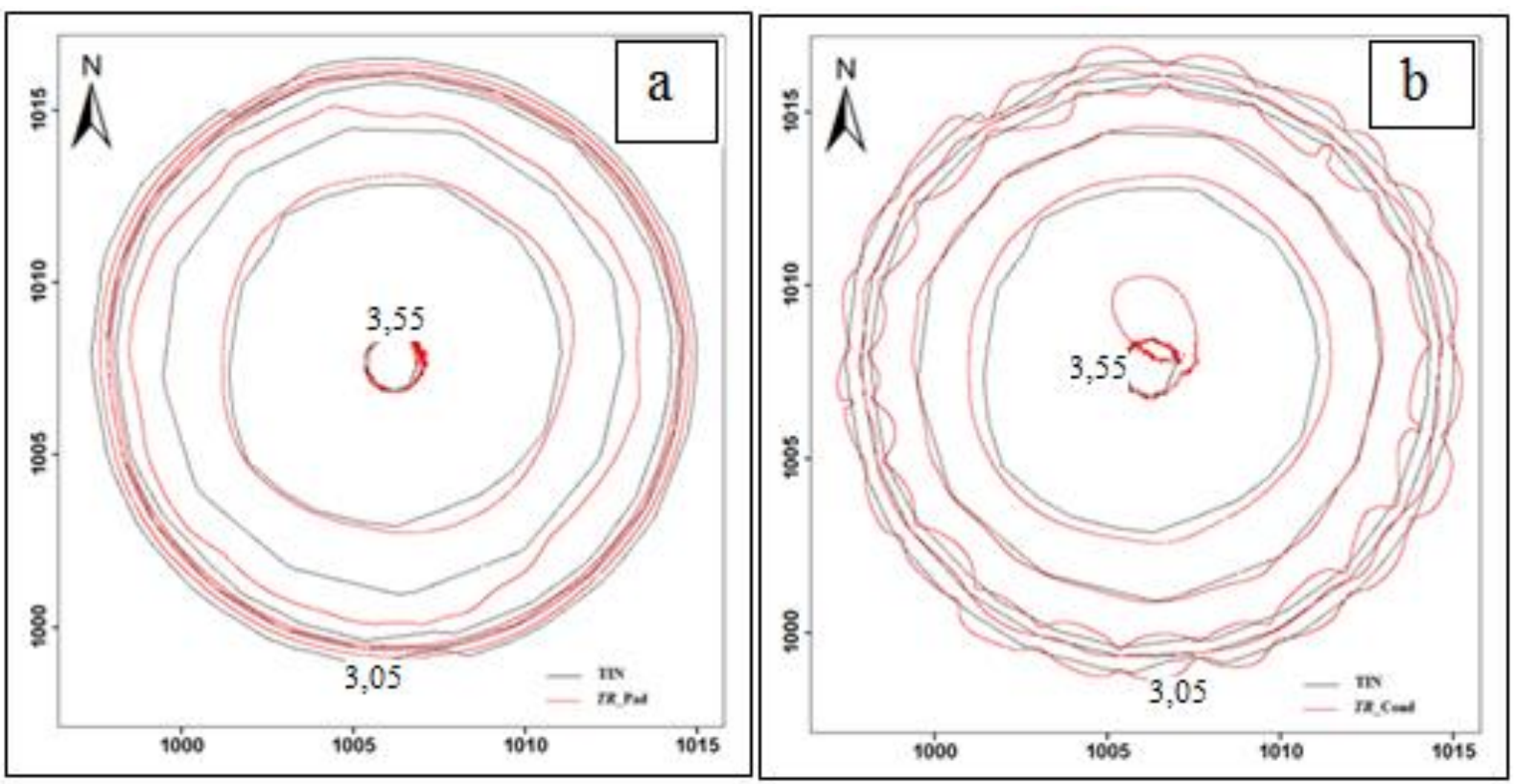

Figura 9: Na cor preta, isobatimétricas geradas pelo método TIN, e em vermelho, pelo Topo to Raster padrão (Figura 9-a) e pelo Topo to Raster condicionado (Figura 9-b).

Bol. Ciênc. Geod., sec. Artigos, Curitiba, v. 21, noำ 4, p.674-690, out-dez, 2015. 
Analisando isobatimétricas na Figura 9, verifica-se que a interpolação empregando Topo to Raster padrão (Figura 9-a) mostrou-se mais realista que Topo to Raster condicionado (Figura 9b), que apresentou irregularidades nas isobatimétricas próxima da borda e do centro. Quando comparada com os métodos de krigagem, (Figura 7), verifica-se também que as isobatimétricas geradas com o Topo to Raster foram mais suavizadas. Vale salientar que a interpretação visual das isobatimétricas foi possível pelo fato de o objeto levantado ser uma figura regular construída em concreto liso.

Os índices calculados para a análise estatística das discrepâncias entre os valores das profundidades estimados e encontrados pelo TIN, se encontram na Tabela 2 para a krigagem ordinária (KO), krigagem simples (KS), Topo to Raster padrão (TR_Padrão) e Topo to Raster condicionado (TR_Condicionado).

Para profundidades médias de 3,21 m, foram encontrados, com os dois métodos, os seguintes valores para os indicadores estatísticos: discrepância máxima de $10 \mathrm{~cm}$, discrepância média de 1 $\mathrm{cm}$, desvio padrão da amostra de $3 \mathrm{~cm}$ e RMS das discrepâncias de $3 \mathrm{~cm}$.

Tabela 2: Índices estatísticos das discrepâncias.

\begin{tabular}{|c|c|c|c|c|c|}
\hline & $\mathrm{RMS}_{\mathrm{d}}(\mathbf{m})$ & $\bar{d}(\mathbf{m})$ & Sd (m) & Max (m) & $\operatorname{Min}(\mathbf{m})$ \\
\hline $\mathrm{KO}$ & 0,032 & 0,006 & 0,032 & 0,088 & $-0,094$ \\
\hline $\mathrm{KS}$ & 0,032 & 0,005 & 0,032 & 0,086 & $-0,095$ \\
\hline TR_Padrão & 0,026 & $-0,014$ & 0,026 & 0,103 & $-0,046$ \\
\hline$T R$ Condicionado & 0,020 & 0,009 & 0,020 & 0,111 & $-0,089$ \\
\hline
\end{tabular}

Ao analisar a Tabela 2, verifica-se que entre os métodos krigagem ordinária (KO) e krigagem simples (KS) que os valores encontrados quase não se diferem, o que se verificou também na análise das isobatimétricas. Fato semelhante observa-se na análise com o Topo to Raster condicionado (TR_Condicionado) e Topo to Raster padrão (TR_Padrão), embora, as isobatimétricas geradas com o Topo to Raster condicionado tenham apresentado irregularidades nas bordas e no centro do reservatório, como mostra a Figura.9.

A krigagem ordinária é mais utilizada que a krigagem simples por não exigir conhecimento da média sobre a área em estudo. Sendo assim assume-se que a krigagem ordinária tenha apresentados resultados mais confiáveis (Andriotti, 1986).

Os resultados encontrados para os RMS das discrepâncias, embora um pouco menores para o Topo to Raster, apresentaram pequenas variações, dificultando a escolha do melhor método. Sendo assim, optou-se então por aplicar a estatística inferencial na média encontrada. Na estatística inferencial a análise é feita através do teste $\mathrm{t}$ de Student ou teste z, sendo que o teste t de Student exige que a amostra siga uma distribuição normal ou gaussiana (Santos, 2010). A escolha do teste se dá a partir do tamanho da amostra. Para amostras menores que 30 pontos deve se utilizar o teste t e para amostras maiores o teste $z$ (Montgomery e Runger, 2008).

Como a amostra possui mais de 30 pontos, aplicou-se o teste z, conforme Equação 13. Onde, $\mu$ é a média esperada, que tem o valor igual a zero. 


$$
\text { zcal }=\frac{\overline{\mathrm{d}}-\mu}{\frac{\mathrm{Sd}}{\sqrt{\mathrm{nd}}}},
$$

A Tabela 3 apresenta os resultados do teste z, mostrando que dentro de um nível de confiança de 95\%, a média das discrepâncias dos interpoladores KO e KS não é significativa, ou seja, não diferem do TIN. Já os interpoladores TR_Padrão e TR_Condicionado apresenta diferença significativa na média quando comparados ao TIN.

Tabela 3: Estatística inferencial para o teste $z$.

\begin{tabular}{c|c}
\hline & $\boldsymbol{z c a l}$ \\
\hline $\mathrm{KO}$ & $1,837^{\mathrm{ns}}$ \\
\hline $\mathrm{KS}$ & $1,531^{\mathrm{ns}}$ \\
\hline$T R \_$Padrão & $5,276^{*}$ \\
\hline$T R \_$Condicionado & $4,409^{*}$ \\
\hline
\end{tabular}

* significativo; ns não significativo.

\section{Conclusões e Recomendações}

Com este estudo pode-se verificar a acurácia dos Modelos Digitais de Elevação de pequenas superfícies, levantadas por topografia, gerados por krigagem ordinária, krigagem simples, Topo to Raster padrão e Topo to Raster condicionado comparando-os com o modelo de elevação gerado por Rede de Triângulos Irregulares - TIN.

Para profundidades médias de $3,21 \mathrm{~m}$, foram encontrados os seguintes valores para os indicadores estatísticos: discrepância média de $1 \mathrm{~cm}$, discrepância máxima de $10 \mathrm{~cm}$, desvio padrão da amostra de $3 \mathrm{~cm}$ e RMS das discrepâncias de $3 \mathrm{~cm}$.

A partir das isobatimétricas verificou-se que o interpolador Topo to Raster condicionado, cujos dados de entrada são somente os pontos cotados, apresentou notáveis deformações nas bordas e no centro da área de estudo, devendo ser descartado.

Aplicando o teste z, conclui-se que os interpoladores krigagem e Topo to Raster padrão, aplicados na interpolação de pequenas superfícies, se diferem do TIN.

Vale salientar que para empregar o Topo to Raster padrão há necessidade de se fornecer como entrada informações de contorno enquanto para krigagem bastam os pontos cotados.

\section{AGRADECIMENTOS}

Ao Departamento de Engenharia Civil - Setor de Engenharia de Agrimensura e Cartográfica da Universidade Federal de Viçosa - UFV pelo apoio a esta pesquisa. 
A Divisão de Água e Esgoto da UFV por colaborar com local de pesquisa.

Ao corpo de bombeiros da UFV por sempre atender aos pedidos solicitados para auxiliar nos trabalhos de campo.

\section{REFERÊNCIAS BIBLIOGRÁFICAS}

Burrough, P.A.; Mcdoneel, R. Priciples of Geographical Information Systems. Spatial information systems and geostatistics. New York: Oxford University Press, 1998.

Camargo, E.C. G. Geoestatística: fundamentos e aplicações. Geoprocessamento para projetos ambientais. São José dos Campos: INPE, 1998.

Carvalho, Q. V. Estudo da Dispersão de Metais Pesados na Envolvente de uma Central Termoelétrica a Carvão. Dissertação de mestrado., Faculdade de Engenharia da Universidade do Porto, Porto, Portugal, 2012.

Chaves, M. A. Modelos Digitais de Elevação Hidrologicamente Consistentes para a Bacia Amazônia. Tese de doutorado., Universidade Federal de Viçosa - UFV, Viçosa, 2002.

Ferreira, I.O; Rodrigues, D.D; Santos, A. de P. Levantamento batimétrico automatizado aplicado à gestão de recursos hídricos. Estudo de caso: Represamento do ribeirão São Bartolomeu, Viçosa-Mg. In: Simpósio Brasileiro de Ciências Geodésicas e Tecnologias da Geoinformação, 4., Recife. Anais.Recife, 2012.

Guimarães, E. C. Geoestatistica Básica e Aplicada. UFU/FAMAT. Núcleo de estudos estatísticos e Biométricos. 2004.

Hutchinson, M. F. A new procedure for gridding elevation and stream line data with automatic removal of spurious pits. Journal of Hydrology, 106, 1989.

Hutchinson, M. F. Calculation of hydrologically sound digital elevation models. Proceedings, Third International Symposium on Spatial Data Handling, Sydney, Columbus: International Geographical Union, 1988.

Hutchinson, M. F. Locally adaptive gridding of noisy high resolution topographic data. XVIII Congresso Mundial IMACS e MODSIM09 Congresso Internacional sobre Modelagem e Simulação. Cairns, Austrália, 2009.

Isaaks, E.H.; Srisvastava, R.M. An Introduction to Applied Geostatistics. New York: Oxford University Press, 1998.

Kamimura, K. M. et al. Load bearing capacity of a Red-Yellow Latosol in a coffee plantation. Revista Brasileira de Ciência do Solo, 36, 2012.

Landim, P. M. B. Introdução aos métodos de estimação espacial para confecção de mapas. DGA, IGCE, UNESP/Rio Claro, Lab. Geomatemática, Texto Didático, 2, 2000.

Landim, P. M. B.; Monteiro, R. C.; Corsi, A. C. Introdução à confecção de mapas pelo software SURFER. DGA, IGCE, UNESP/Rio Claro, Laboratório Geomatemática, Texto Didático, 8, 2002.

Mesquita, S. M. P. Modelação da distribuição espacial da qualidade do ar em Lisboa usando sistemas de informação geográficas. Dissertação de mestrado., Instituto Superior de Estatística e Gestão de Informação. Universidade Nova de Lisboa. Lisboa. Portugal, 2009. 
Montgomery, D. C.; Ruger, G.C., Estatística aplicada e probabilidade para engenheiros. Tradução Verônica Calado, 2, Rio de Janeiro: LTC, 2008.

Nogueira, J. D. L. de; Amaral, R. F. do. Comparação entre os métodos de interpolação (krigagem e Topo to Raster) na elaboração da batimetria na área da folha Touros-RN. XIV Simpósio Brasileiro de Sensoriamento Remoto, 2009.

Pinto, S. Um Sistema de Representação de Relevos. Dissertação de mestrado., DCC/ICEX/UFMG, Belo Horizonte, 1994.

Santos, A.P. Avaliação da acurácia posicional em dados espaciais com uso de estatística espacial. Dissertação de mestrado., Universidade Federal de Viçosa - UFV, Viçosa, 2010.

Santos, G.R., et al. krigagem simples versus krigagem universal: qual o preditor mais preciso?. Energia na Agricultura, 26, 2011.

Schaffrath, V.R. Variabilidade espacial de propriedades físicas do solo e de variáveis de plantas daninhas em sistemas de manejo de solo. Tese de doutorado., Universidade Estadual do Paraná, Maringá, 2006.

Soares, A. Geoestatística para as Ciências da Terra e do Ambiente. Lisboa, 2, 2006.

Tobler, W. R. A computer movie simulating urban growth in the Detroit region. Economic Geography, 1970.

Vilela, L. C. Comparação entre as redes neurais artificiais e o método de interpolação krigagem aplicados à pesquisa agronômica. Tese de doutorado., UNESP. Faculdade de Ciências Agronômicas, 2004.

Yamamoto, J. K; Conde, R. P. Classificação de Recursos Minerais Usando a Variância de Interpolação. Brazilian Journal of Geology, 29, 1999.

Recebido em Outubro de 2014.

Aceito em Abril de 2015. 